\title{
The Effects of a Pool of Dispersers on Host-parasitoid Systems
}

\author{
Wolfgang W. Weisser, $* \$$ Vincent A. A. Jansen† $\uparrow$ and Michael P. Hassell* \\ *Department of Biology, and the $\dagger N E R C$ Centre for Population Biology, \\ Imperial College at Silwood Park, Ascot, Berks. SL5 7PY, U.K.
}

(Received on 24 March 1997, Accepted in revised form on 8 August 1997)

\begin{abstract}
When individuals migrate in a multi-patch environment, a considerable proportion of their lifetime might be spent in transit between patches. We investigate the effects such a pool of dispersers can have on local stability and dynamics for a variety of multi-patch host-parasitoid models. When an arbitrary number of patches with internal Lotka-Volterra dynamics is coupled via a global pool of dispersers, the equilibrium is globally stable. The global pool is stabilising if dispersal is by hosts only, by parasitoids only, or by both hosts and parasitoids. If dispersal is local such that individuals first enter a pool close to the patch where they originate and then disperse to adjacent pools, the equilibrium is locally stable. We also analyse the situation where the functional response of parasitoids within a patch is Holling type II which is known to destabilise host-parasitoid systems. Coupling this single patch to a pool of dispersers can produce a locally stable interaction, provided the handling time of hosts is not too long. However, the pool provides a biologically realistic example of an interaction that is locally stable but not permanent. The longer the handling time, the smaller the region of population densities within which populations converge to the equilibrium state. In a multi-patch environment with a global disperser pool, the dynamics of the system are not qualitatively different from the single patch case (i.e. the equilibrium can be locally stable but the system is not permanent). In a multi-patch environment with local disperser pools, true spatial interactions between patches can develop. In contrast to the global pool, local pools can destabilise the stable equilibrium of the single patch case. Limit cycles develop around this unstable equilibrium that lead to extremely complicated dynamics. In contrast to the global pool, a system of local pools can exhibit bounded fluctuations so that populations do not go extinct.
\end{abstract}

(C) 1997 Academic Press Limited

\section{Introduction}

In recent years there has been an extensive interest in the effects of dispersal on the population dynamics of predator-prey or host-parasitoid systems (Hassell et al., 1991a, b; Holt, 1984, 1985, 1993; Jansen, 1994, 1995a, b; Murdoch, 1994; Reeve, 1988, 1990; Taylor, 1990, 1991; Weisser \& Hassell, 1996). A major

\$Author to whom correspondence should be addressed. §Present address: Zoology Institute, Rheinsprung 9, CH-4051 Basel, Switzerland.

TPresent address: Mathematical Biology Group, Department of Zoology, University of Oxford, South Parks Road, Oxford OX1 3PS, U.K. impetus for this research were the early observations by Huffaker (1958) and Pimentel et al. (1963) that interacting populations of predators and prey or of hosts and parasitoids would persist longer when individuals had to disperse between different resource patches.

Population dynamic models that incorporate dispersal have mainly focussed on the way in which movement distributes individuals in space. For example, aggregation of parasitoids in certain host patches can result in a skewed distribution in the risk of a host of being parasitized, which has been shown to have a stabilising effect in a wide range of non-spatial host-parasitoid models (Sabelis \& 
Diekman, 1988; Pacala et al., 1990; Hassell et al., 1991a, b). In metapopulation models, spatial heterogeneity in any of the populations' vital parameters (birth, death, immigration and emigration rates) can result in asynchronous population oscillations so that the populations can persist on a regional scale even when local interactions are unstable (McLaughlin \& Roughgarden, 1993; Reeve, 1988, 1990). Here, the reason for persistence is that the displacement of individuals prevents diverging oscillations.

Apart from displacing individuals in space, however, a further consequence of dispersal is the absence of possibly a large number of individuals from patches at any one time. Depending on the spacing of resources, individuals spend different amounts of time in transit between patches. The longer the distances between patches, the higher the proportion of an individual's life-time that may be spent searching for a new host patch. For both hosts and parasitoids, travelling between patches decreases the time available for breeding activities. Furthermore, travelling between patches entails the risk that an animal might die before a new patch is reached. Although data of insect dispersal in the field are generally scarce, studies such as the one by Driessen \& Hemerik (1992) on dispersal of the Drosophila parasitoid Leptopilina clavipes suggest that travel times can cause a high proportion of individuals to be in transit between patches at any one time (ca. 48\% in Driessen \& Hemerik's most realistic scenario). The effects of travel time and travel mortality have mainly been investigated in models of animal foraging behaviour (e.g. Charnov, 1976; Houston \& McNamara, 1986; Weisser et al., 1994). Population models have focussed on the way travel time changes the distribution of animals over patches (e.g. Hassell \& May, 1974; Weisser et al., 1997) or the way travel mortality increases the death rates of a population (Ruxton et al., 1997).

The existence of a "pool" of migrants, in which individuals spend some time before they find empty patches, has been addressed in only a few studies of single species (Gyllenberg \& Hanski, 1992) and predator-prey systems (Metz \& Diekman, 1986; Diekman et al., 1988; Sabelis et al., 1991; Jansen \& Sabelis, 1992; Jansen, 1994, 1995a; Rinaldi et al., 1996). In this paper, we investigate the effects of a pool of dispersers in a variety of multi-patch models. Our approach differs from the previous studies in that we concentrate on the effect of dispersal in a system where local populations do not go extinct so that there is no recolonisation of empty patches. Weisser \& Hassell (1996) showed that a pool of dispersers can stabilise the simple Lotka-Volterra model. In this paper, we extend these results further. We first consider individuals that emigrate from patches into a "global" disperser pool from which they are distributed evenly over patches [Fig. 1(a)], implying that dispersers from a patch can reach all patches equally well. In this case it is assumed that mixing is complete in the global pool so that the formation of any spatial patterns from local interactions between patches is prevented. We then test the robustness of our results and study the case where dispersal is local, i.e. individuals leaving patches disperse into a local pool, around the patch from which they originate [Fig. 1(b)]. For both scenarios, we first investigate the effects of a pool of dispersers for the case of simple Lotka-Volterra dynamics within patches. In a second step, we analyse the case when the functional response of parasitoids within patches is Holling type II, which is known to destabilise the local interaction. This allows us to investigate the strength of the stabilising effect of a pool of dispersers.

(a)

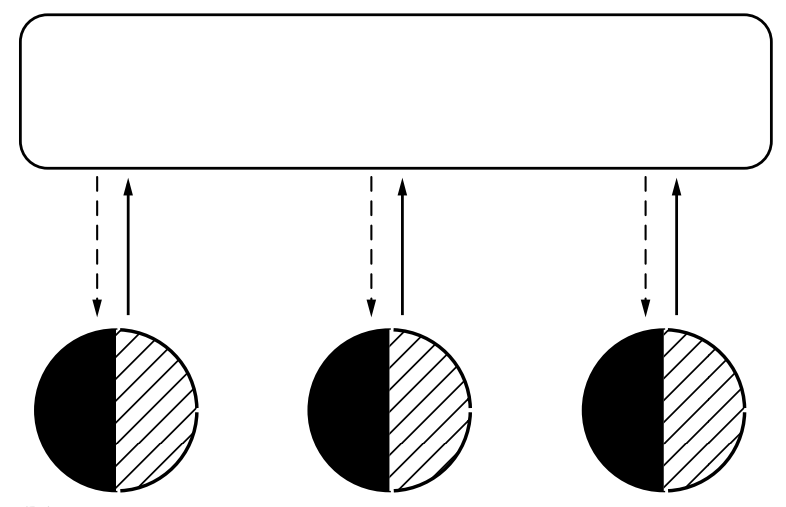

(b)

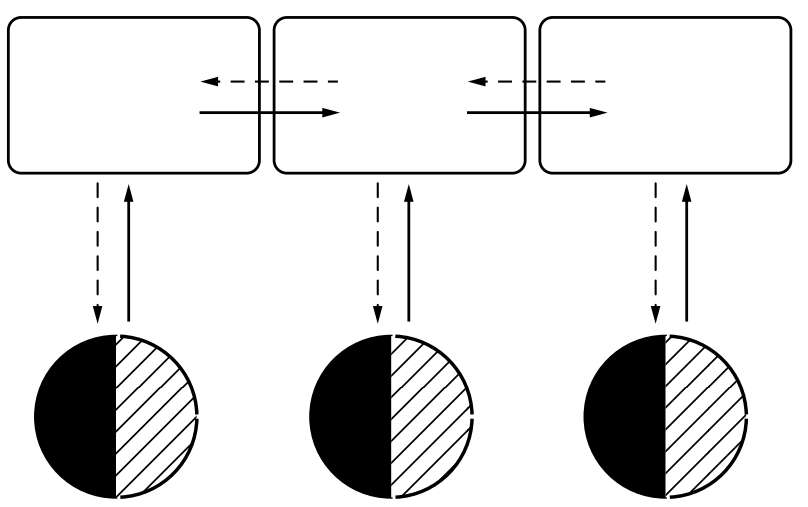

FIG. 1. Illustration of patches connected by a global pool (a) and patches connected by local pools (b). 


\section{Dispersal in a Multi-patch Environment}

We model host and parasitoid dynamics for an environment that consists of $n$ patches. Let $H_{j}$ and $P$ denote the densities of hosts and paratoids, respectively, that are in patch $j$ at time $t$. Within each patch the dynamics are given by simple LotkaVolterra equations. Dispersal of host and parasitoids is independent of the density of animals in the patch. Hosts leave a patch at rate $\varepsilon$ and parasitoids disperse from patches at rate $e$. We assume that all travelling hosts and parasitoids enter a single (global) pool of dispersers from which they are re-distributed equally among patches [Fig. 1(a)]. Let $R$ and $Q$ denote the densities of hosts and parasitoids, respectively, that are in transit between patches at time $t$. In the host pool, let $\sigma$ denote the rate at which hosts are lost from the system because they do not survive the journey from one patch to another. Similarly, $s$ denotes the death rate for migrating parasitoids. Immigration of individuals into patches is independent of the density of individuals in the pool. Hosts immigrate into a particular patch with rate $\eta$, and parasitoids with rate $i$. With these assumptions, we arrive at the following set of equations:

$$
\begin{gathered}
\dot{H}_{j}=r H_{j}-H_{j} P_{j}-\varepsilon H_{j}+\eta R \\
\dot{P}_{j}=H_{j} P_{j}-d P_{j}-e P_{j}+i Q \\
\dot{R}=\varepsilon \sum_{j=1}^{n} H_{j}-m \eta R-\sigma R \\
\dot{Q}=e \sum_{j=1}^{n} P_{j}-n i Q-s Q .
\end{gathered}
$$

Here, $r$ denotes the rate at which hosts grow in the absence of parasitoids. The rate at which hosts are parasitized within a patch $j$ is proportional to the local density $P_{j}$, i.e. we assume a linear functional response; the attack rate is scaled to unity. The number of hosts emerging from a parasitized host is assumed to be one (solitary parasitoids), and the parasitoid death rate within patches is given by $d$.

Without dispersal $(e=\varepsilon=0)$ and immigration $(\eta=i=0)$, the system reduces to a number of uncoupled patches of which the dynamical properties are well-known (Volterra, 1926). The parasitoid equilibrium density $P^{*}=r$, depends on the host growth rate, and the host equilibrium density $H^{*}=d$ is dependent upon the parasitoid death rate. This equilibrium is neutrally stable so that after disturbance, population densities do not converge back to the equilibrium densities. (In the following, we use the term "stable" for locally asymptotically stable. With stabilising we mean the transition from instability or neutral stability to asymptotic stability.)

If either hosts or parasitoids disperse from a single patch and form a pool of migrants, the LotkaVolterra system is stabilised (Weisser \& Hassell, 1996). We now analyse the stability properties of the multi-patch system (1) where the population dynamics in each patch are modelled explicitly. This system has an equilibrium in which the parasitoids and hosts in all patches have identical densities, $P^{*}$ and $H^{*}$, respectively. The equilibrium densities of the pools are given by

$$
Q^{*}=\frac{n e}{n i+s} P^{*} \text { and } R^{*}=\frac{n \varepsilon}{n \eta+\sigma} H^{*} .
$$

Hense the equilibrium densities of animals within patches are:

$$
\begin{aligned}
& H^{*}=d+e-\frac{n e i}{n i+s}=d+\frac{e s}{n i+s} \\
& P^{*}=r-\varepsilon+\frac{\varepsilon n \eta}{n \eta+\sigma}=r-\frac{\varepsilon \sigma}{n \eta+\sigma}
\end{aligned}
$$

The equilibrium is positive provided

$$
r>\frac{\varepsilon \sigma}{n \eta+\sigma}
$$

Thus, for a given number of patches and a particular combination of host emigration, immigration and pool death rates there is a minimum value for the intrinsic growth rate for the hosts. A low immigration rate $\eta$, a high mortality rate during travelling, $\sigma$, or a emigration rate, $\varepsilon$, all result in a higher intrinsic growth rate necessary for a positive equilibrium. Alternatively, an increase in the number of patches in the environment lowers the minimum value for the host growth rate. If the number of patches is sufficiently large, the equilibrium will be positive even for very small values of $r$. In Appendix A we show that the equilibrium is unique and globally stable in the first orthant, i.e., after any pertubation that keeps population densities strictly positive the population densities will return to the equilibrium values. Thus, in a true multi-patch environment, the absence of a fraction of individuals from patches at any one time can stabilise host-parasitoid systems. Provided the conditions concerning the host growth rate are fulfilled, very small rates of dispersal can stabilise the interaction between host and parasitoids. In Appen$\operatorname{dix} \mathrm{A}$ it is shown that the system remains globally stable when either hosts and parasitoids are sessile and do not disperse, provided that initially the 
numbers of the sessile species are positive in all patches. It is important to note that dispersal here is not biased with respect to where individuals migrate to in the environment, and that dispersal is not density-dependent. Thus, there is no aggregation of parasitoids in patches and consequently no "aggregation of risk" (Chesson \& Murdoch, 1986; Hassell et al., 1991a) among host-individuals within patches.

\section{A Multi-patch Environment Where Dispersal is Local}

In the above section, we considered the case where individuals disperse into a global pool and were re-distributed equally over all patches in the habitat. This scenario implies that dispersers that leave the pool are as likely to immigrate into a patch far away from the one where they originated as they are to immigrate into a patch very close to their original patch. An alternative scenario is to assume that dispersal is mainly local; i.e., that individuals that emigrate from a patch first enter a local pool around their original patch [Fig. 1(b)]. The migrants that have dispersed into this local pool then either return to the same patch or they migrate further into an adjacent pool. From there, they might enter the patch close to the new pool or continue migrating, either back to the pool where they came from or into another adjacent pool. In this scenario, therefore, migrants are much more likely to return to patches close to the patch where they originated than to patches that are further away. In species where dispersal abilities are limited, such a system of local pools might describe the biological situation better than a model with a global disperser pool (note that we still assume that dispersal is undirected and that dispersal rates are independent of animal densities in either patches or pools).

The multi-patch model with local disperser pools for hosts and parasitoids has the following dynamics:

$$
\begin{gathered}
\dot{H}_{j}=r H_{j}-H_{j} P_{j}-\varepsilon H_{j}+\eta R_{j} \\
\dot{P}_{j}=H_{j} P_{j}-d P_{j}-e P_{j}+i Q_{j} \\
\dot{R}_{j}=\varepsilon H_{j}-(\eta+\sigma) R_{j}+m_{r} \sum_{i=1}^{n} c_{i j} R_{i} \\
\dot{Q}_{j}=e P_{j}-(i+s) Q_{j}+m_{q} \sum_{i=1}^{n} c_{i j} Q_{i} .
\end{gathered}
$$

Here, host dispersal from patch $i$ to patch $j$ pools is given by $m_{r} c_{i j} R_{i}$ where $m_{r}$ is the standard host migration rate and $c_{i j}$ is the relative importance of the migration from patch $i$ to patch $j$. All $c$ s form the matrix $\mathbf{C}=\left\{c_{i j}\right\}$ which describes how the patches are connected. For instance, a chain of patches has a $\mathbf{C}$ of the following form:

$$
\left(\begin{array}{rrrrrrr}
-1 & 1 & 0 & \ldots \ldots \ldots \ldots \ldots \ldots \ldots \ldots \ldots & 0 \\
1 & -2 & 1 & 0 & \ldots \ldots \ldots \ldots \ldots \ldots . & 0 \\
0 & 1 & -2 & 1 & 0 & \cdots \ldots . & 0 \\
. & \ldots & \ldots & \ldots & \ldots & \cdots & . \\
0 & \ldots & 0 & 1 & -2 & 1 & 0 \\
0 & \ldots \ldots \ldots \ldots \ldots \ldots & 0 & 1 & -2 & 1 \\
0 & \ldots \ldots \ldots \ldots \ldots \ldots \ldots \ldots \ldots & 0 & 1 & -1
\end{array}\right)
$$

Parasitoid dispersal between pools is given by $m_{q} c_{i j} Q_{i}$ where $m_{q}$ is the standard migration rate of parasitoids between pools. Appendix B shows that the equilibrium of system (3) is stable for the simplest case of two patches connected by local pools. From this it follows that the system is stable for any number of patches connected by local pools (Jansen \& de Roos, 1998; Jansen, 1998). Thus, if patches are connected by local pools of dispersers, these local pools also stabilise the Lotka-Volterra model.

To summarise, if hosts or parasitoids emigrate from patches and enter a pool of dispersers before they return to patches, this has a stabilising effect on the simple Lotka-Volterra model. A pool of dispersers is stabilising both in the case of global dispersal of individuals as well as in the case of dispersal into local pools adjacent to the patches. This suggests that spatial patterns of local abundance are not possible under Lotka-Volterra dynamics [see also Murray, (1975)].

\section{A Destabilising Functional Response}

In above sections, we showed that a system of patches can be stabilised by coupling these patches via a pool of migrants. We now analyse the effects of a pool of dispersers in a more complex system. We assume that within patches, parasitoids are limited in the number of hosts they can parasitise so that they show a Holling type II functional response with respect to host density (Rosenzweig \& McArthur, 1963; Hassell, 1978). This functional response is known to destabilise host-parasitoid systems. Adding a Type II functional response causes populations in isolated patches to show diverging oscillations. To simplify our considerations, we first consider the case 
of a single patch connected to a pool. The functional response of the parasitoids is now given by:

$$
\frac{H}{1+T_{h} H} \text {. }
$$

Here, $T_{h}$ describes the handling time of the parasitoids. If we ignore host dispersal, the systems dynamics can be described as:

$$
\begin{gathered}
\dot{H}=r H-\frac{H P}{1+T_{h} H} \\
\dot{P}=\frac{H P}{1+T_{h} H}-(d+e) P+i Q \\
\dot{Q}=e P-(i+s) Q .
\end{gathered}
$$

The equilibrium values are given by:

$$
\begin{aligned}
Q^{*} & =\frac{e}{i+s} P^{*} \\
P^{*} & =\frac{r}{1-\alpha T_{h}} \\
H^{*} & =\frac{\alpha}{1-\alpha T_{h}}
\end{aligned}
$$

where

$$
\alpha=d+\frac{\mathrm{es}}{\mathrm{i}+\mathrm{s}},
$$

note that this is the prey equilibrium of system (1). The equilibrium is positive if $T_{h}<1 / \alpha$. Thus, the longer the handling time of the parasitoids, the less likely it is that the equilibrium is positive. An increase in the parasitoid death rate within patches, $d$, the parasitoid death rate while travelling, $s$, or the parasitoid emigration rate from patches, $e$, make a positive equilibrium less likely. On the other hand, increasing the immigration rate into patches, $i$, facilitates a positive equilibrium. Appendix C analyses the stability of this equilibrium. The equilibrium is locally stable if

$$
\begin{gathered}
T_{h}<(d+e+i+s)^{-1} \\
\text { and } \\
r \alpha<\frac{1}{T_{h}}\left(i+s+\frac{i e}{i+s}-\frac{\left(1-\alpha T_{h}\right)(i+s)}{1-T_{h}(d+e+i+s)}\right)
\end{gathered}
$$

Thus, the longer the handling time, the smaller the range of parameter-values for which an equilibrium is locally stable. Holt (1985) has previously analysed the stability properties of system (5), where, although formally equivalent, the model was used with a slightly different interpretation. The dynamic properties of system (5) are illustrated in Fig. 2. These figures were obtained by using automated bifurcation analysis (Khibnik et al., 1993). Figure 2 gives the host equilibrium density and the limit cycles as a function

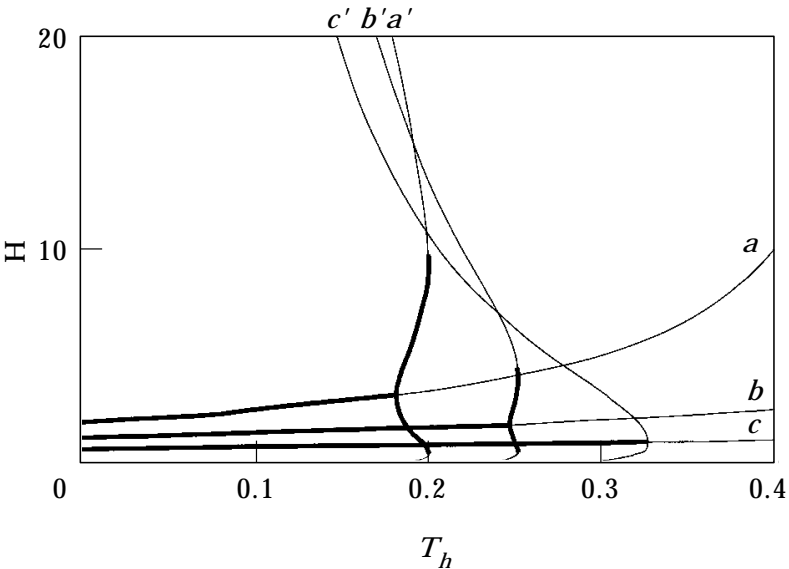

Fig. 2. Host equilibrium density $H^{*}$ and limit cycles of system (5) as a function of handling time $T_{h}$ for three values of $\alpha$. Thick lines represent stable equilibria, thin lines unstable equilibria. Parameter values. $r=1, e=1, i=s=0.2$, for the curve labelled $a: d=1.5(\alpha=2)$, the curve labelled $b: d=0.75(\alpha=1.25)$, and the curve labelled $c: d=0.25(\alpha=0.75)$. The curves labelled $a^{\prime}, b^{\prime}$ and $c^{\prime}$ represent the maximum and minimum of the host density $H$ over the limit cycle for the corresponding parameter values. The thicker part of these curves represents stable limit cycles, the thinner parts unstable limit cycles.

of $T_{h}$. For $T_{h}=0$ (Lotka-Volterra case), there is no limit cycle and the system is globally stable. This is the situation analysed by Weisser \& Hassell (1996). Figure 2 suggests that for $T_{h}>0$, the locally stable equilibrium is surrounded by an unstable limit cycle. The amplitude of the limit cycle decreases as the handling time increases and the limit cycle approaches the equilibrium point towards the Hopf bifurcation. In effect, the existence of an unstable limit cycle around the equilibrium means that stochasticity of sufficient magnitude would cause populations to leave the region in which densities converge back to the equilibrium point. Once the densities have left the domain of attraction of the stable equilibrium the populations show diverging oscillations leading to the extinction of both hosts and parasitoids.

Figure 2 shows that in a limited region of parameter-space, the locally unstable equilibrium is surrounded by a stable limit cycle. The limit cycle is stable before it folds back into an unstable limit cycle. In the region in parameter space where the unstable equilibrium is surrounded by a stable limit cycle, the host-parasitoid association can oscillate without becoming extinct. However, the stable limit cycle is always surrounded by an unstable limit cycle so that again added stochasticity of sufficient magnitude would cause the populations to become extinct. Figure 2 also shows an example where there is only an unstable limit cycle around the equilibrium. From our simulations it appears that the regions of parameter space for which we find stable limit cycles 
are rather small so that the dynamic behaviour of the model is mostly dictated by the stable equilibrium.

Figure 2 illustrates the difference between local stability and permanence (uniform persistence) of interactions between species. Permanence implies that if all species are present initially, even if only at low densities, then after some time a sizeable amount of each will be present (Hofbauer \& Sigmund, 1988). For our system, the pool can cause local stability of the equilibrium point, but the interactions between hosts and parasitoids are not permanent, as perturbations might bring the population densities into a region where divergent oscillations will cause the extinction of the populations.

In the next section, we extend this single-patch model to a multi-patch model, both for the case of dispersers emigrating into a global disperser pool and for the case of local dispersal. We analyse if this increase in spatial complexity extends the regions of parameter space where the populations can coexist.

\section{A Multi-patch Model with a Destabilising Functional Response}

Spatial interactions can bound fluctuations in a coupled Lotka-Volterra model, even in the absence of density-dependent processes (Jansen, 1995b). Potentially, a coupling of two or more patches with a type II functional response could lead to solutions in which the interactions between the patches keep the densities from going to infinity. We first consider the case of the global disperser pool. A system of two identical patches coupled by a single pool of dispersing parasitoids, $Q$, is given by $(j=1,2)$,

$$
\begin{gathered}
\dot{H}_{j}=r H_{j}-\frac{H_{j} P_{j}}{1+T_{h} H_{j}} \\
\dot{P}_{j}=\frac{H_{j} P_{j}}{1+T_{h} H_{j}}-(d+e) P_{j}+i Q \\
\dot{Q}=e \frac{P_{1}+P_{2}}{2}-(i+s) Q .
\end{gathered}
$$

In this notation, $Q$ is the average number of dispersers per patch in the pool so that the total number of dispersers is $2 Q$. We chose this form of notation for the pool because it facilitates a comparison with the one-patch model analysed above. One equilibrium of the system is given by $H_{1}^{*}=H_{2}^{*}=H^{*}$, $P_{1}^{*}=P_{2}^{*}=P^{*}, Q=Q^{*}$; i.e., the densities of hosts and parasitoids in both patches are identical. Local stability analysis (Appendix D) of the equilibrium shows that the equilibrium is stable under conditions (7) that ensured stability for the single patch case. However, this does not exclude the existence of other stable solutions, such as stable limit cycles or other attractors. A possible way in which these could come into existence would be through a symmetry-breaking bifurcation from limit cycles or from equilibria in which all patches have equal densities. Figure 3 shows the results of automated bifurcation analysis (Khibnik et al., 1993) of system (8). This shows that the dynamics of the model are much more complicated than a local stability analysis of the equilibrium would suggest. Although we found symmetry-breaking bifurcations that potentially could lead to persistence of interactions, we did not find a persisting interaction in any of our simulations. Thus, our host-parasitoid association does not become more persistent as we increase the level of spatial complexity.

Finally, we analyse the multi-patch model with a destabilising response when dispersal is local [Fig. 1(b)]. As above, we simplify the considerations by assuming that the hosts are sessile and only the parasitoids disperse. Under these assumptions, the systems dynamics are given by

$$
\begin{gathered}
\dot{H}_{j}=r H_{j}-\frac{H_{j} P_{j}}{1+T_{h} H_{j}} \\
\dot{P}_{j}=\frac{H_{j} P_{j}}{1+T_{h} H_{j}}-(d+e) P_{j}+i Q_{j} \\
\dot{Q}_{j}=e P_{j}-(i+s) Q_{j}+m_{q} \sum_{i=1}^{n} c_{i j} Q_{i} .
\end{gathered}
$$

In Appendix $\mathrm{E}$ the stability properties of the equilibrium a two-patch version of system (9) are analysed. The results can be easily generalised to any multi-patch model, using a recently developed

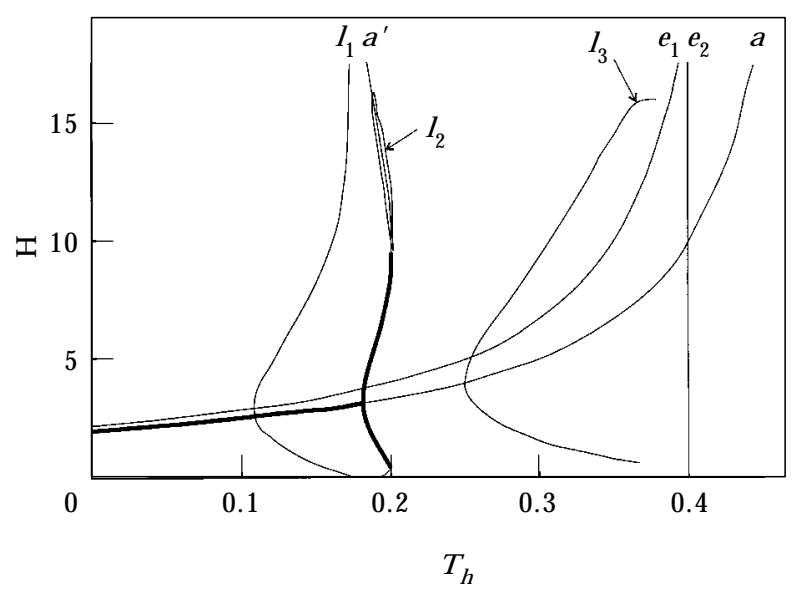

FIG. 3. A partial bifurcation diagram in the predator handling time $T_{h}$ of the multi-patch system with a global pool of dispersers (8). The curves labelled $a$ and $a^{\prime}$ are the same as in Fig. 2, the curves labelled with $e_{i}$ represent equilibria, the curves labelled $l_{i}$ limit cycles. Notice that although the diagram is much more complex, no stable solutions exist other than the ones in Fig. 2. Parameter values as in Fig. 2, curve $a$. 


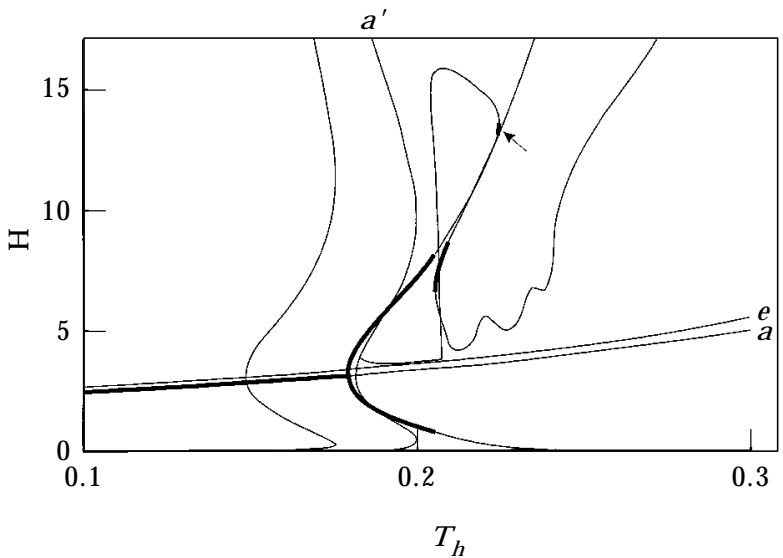

FIG. 4. A partial bifurcation diagram in the predator handling time $T_{h}$ of the multi-patch system with a local pool of dispersers (9). The curves $a$ and $a^{\prime}$ as in Fig. 2, the curve labelled $e$ represents an equilibrium, all unlabelled curves limit cycles. Note that the limit cycle $a^{\prime}$ that was partly stable in Fig. 2 is now unstable but that other stable solutions exist.

method (Jansen \& de Roos, 1998; Jansen, in preparation). In contrast to the situations analysed so far, it is now possible for a locally stable equilibrium to become unstable in the transition from the single patch case to the multi-patch case. The reverse, however, is not true: the unstable equilibrium of the single patch case never becomes stable in the multi-patch case. Figure 4 shows results from our bifurcation analysis. This diagram is even more complicated than the previous one. Interestingly, there are regions of parameter-space where stable spatially inhomogeneous solutions exist. In these regions of parameter-space, spatial interactions prevent the population densities of hosts and parasitoids from going to infinity (and hence extinction). Figure 5 illustrates such a situation where despite local instability the density fluctuations of the populations are bounded. Thus, in the multi-patch case with local pools coexistence of hosts and parasitoids is possible, even for orbits leaving the domain of attraction of an equilibrium. This was not the case in the single patch case [system (5)] where the existence of an unstable limit cycle around the locally stable equilibrium meant diverging oscillations and hence extinction when an orbit left the domain of attraction of the equilibrium.

\section{Discussion}

Models of spatial interactions mainly focus on the outcome of the dispersal process; i.e. the redistribution of individuals in space. Dispersal, however, can also have the effect of introducing time-lags in recruitment. The longer individuals have to travel between patches, the longer the time before they

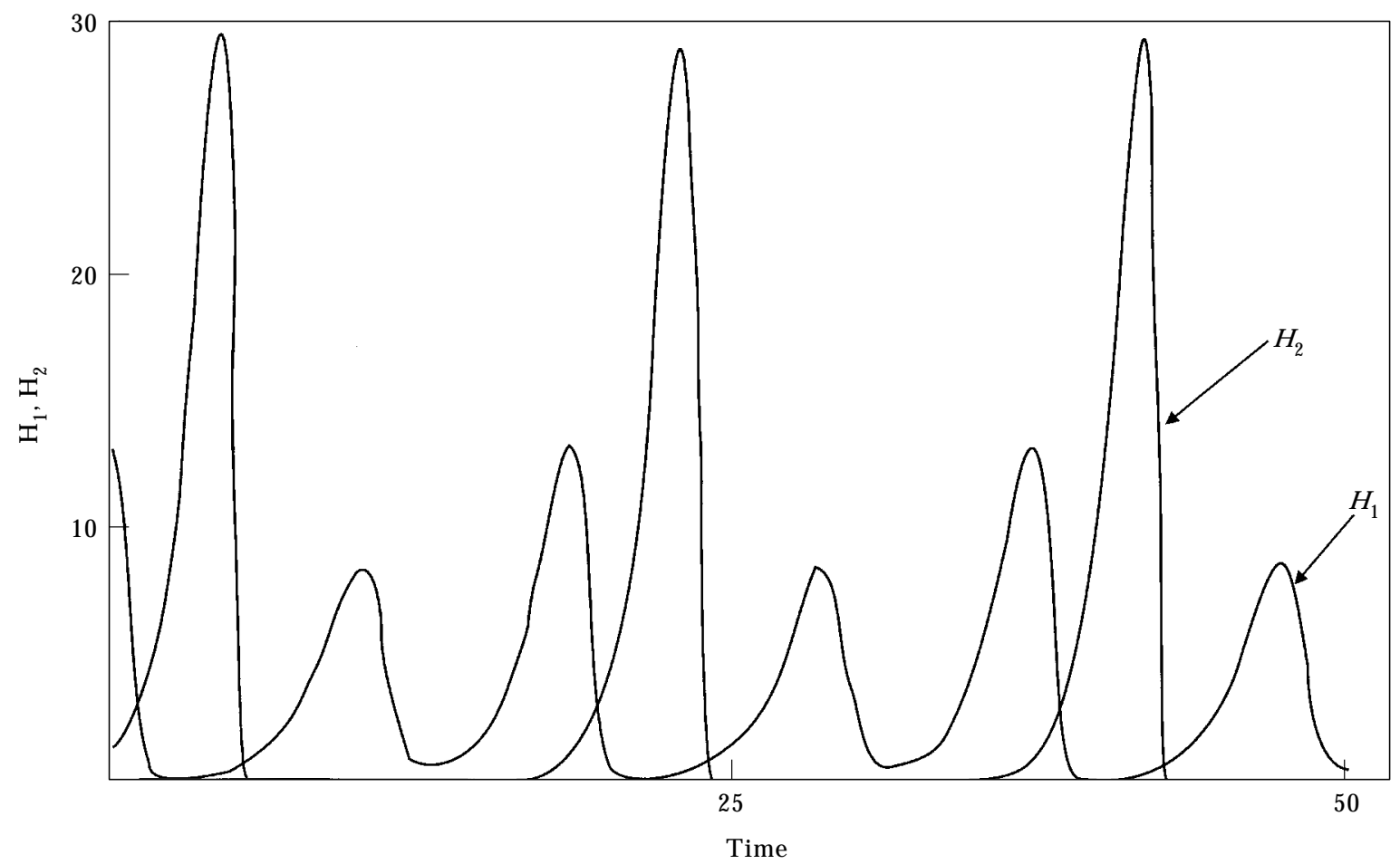

Fig. 5. A run showing bounded fluctuations in a two patch model with local pools (9). In this region of parameter-values the equilibrium is unstable (arrow in Fig. 4). Parameter values as in Fig. 2 curve $a, T_{h}=0.2245, m_{q}=0.1$. 
reproduce in a new patch. For the individuals, the journey from one patch to another also bears costs in terms of mortality risks and time lost for reproduction. While these effects of travelling between patches have been extensively explored in models of animal foraging behaviour (Ayal \& Green, 1993; Houston \& McNamara, 1986; Stephesn \& Krebs, 1986; Weisser et al., 1994) and the evolution of dispersal (e.g. Hamilton \& May, 1977; Hastings, 1983; Holt, 1985), they have been largely neglected in the analysis of metapopulation systems. Ruxton et al. (1997), using a discrete-time framework, show that adding travel mortality to a simple single-species coupled map lattice changes the nature of unstable dynamics. Weisser \& Hassell (1996) found that adding a pool of dispersing individuals can stabilise the LotkaVolterra model. The main aim of the current analysis has been to explore the effects of a pool of dispersers on population dynamics in a more complex setting. Our first result is that a pool induces global stability when a number of patches are coupled to a single (global) pool of dispersers. The dynamics in each patch are described by identical Lotka-Volterra equations, dispersal is random, and no heterogeneity is added to the model. At first sight, this result might seem to contradict previous findings that a coupling of patches without spatial heterogeneity cannot stabilise an unstable equilibrium (e.g. McLaughlin \& Roughgarden, 1993). However, the combination of a number of patches coupled to a pool of dispersers results, in effect, in a source-sink structure where the pool acts as a sink for the individuals. Holt (1984, $1985,1993)$ has analysed the dynamics of predatorprey systems in the context of source-sink systems. Coupling an unstable source to a sink can stabilise a predator-prey system (Holt, 1984, 1985). Holt (1984, 1993) discusses the stabilising effect of a sink in terms of the increased mortality rate experienced by individuals and the time-lagged recruitment of individuals to the source. A pool of dispersers damps the fluctuations in the patches and it introduces heterogeneity in the birth and death rates of the populations. Nevertheless, it has to be emphasized that there is no spatial heterogeneity among any of the population parameters among the patches.

When the functional response of the parasitoids is Holling type II within patches, local dynamics are unstable. The coupling of a such a patch to a pool of dispersing parasitoids can lead to a stable equilibrium. Local stability can be induced by the pool if the handling time of parasitoids is not too long. However, the dynamical behaviour of the system becomes more complex than suggested by linearisation around the equilibrium. In fact, the model provides a good example of the difference between local stability and permanence. The combined effect of the stabilising effects of a pool and the destabilising effects of the functional response are population dynamics which converge to equilibrium if the initial conditions are chosen close to the equilibrium, and which result in increasing fluctuations if the initial conditions are chosen further away from the equilibrium. Thus, a pool of dispersers can stabilise the interaction between hosts and parasitoids, but does not make it permanent. The domain of attraction to the equilibrium goes to infinity in the case of a zero handling time [the situation analysed by Weisser \& Hassell (1996)], and decreases with increasing handling time. Thus, for non-zero handling times, stochasticity of large enough amplitude would always be expected to bring population dynamics into the regions of population densities where violent oscillations causing the extinction of the populations set in. There are regions in parameter-space where stable limit cycles surround the equilibrium. However, if population densities do not fall within their rather small domains of attractions, increasing fluctuations are again the result. These results do not qualitatively change when further patches are added to the model. Although the dynamics become extremely complicated when a system of patches within which the functional response of the parasitoids is Holling type II is coupled to a single pool, no bounded fluctuations were found except the ones where initial population densities were within the domain of attraction of the stable equilibrium or limit cycle. Thus, as far as the persistence of the host-parasitoid interaction is concerned, the metapopulation with a global pool is qualitatively not different from the non-spatial case.

In our second scenario, migrating individuals first disperse into local pools around the patches from which they originate [Fig. 1(b)]. These units, each consisting of a patch and its local pool, are coupled by individuals migrating between adjacent pools. In this scenario, there is an explicit spatial dimension as dispersing individuals are more likely to immigrate into nearby patches than into patches further away. If, within patches, there are Lotka-Volterra dynamics, the stability properties of the multi-patch case (where a number of units are coupled by dispersal) are identical to the stability properties of a single unit. Thus, in the Lotka-Volterra case, no true spatial interactions develop between the patches, despite the spatially explicit set-up. If, in contrast, the functional response of the parasitoids within patches is Holling type II, the stability properties of the multi-patch system differ from the properties of the single-patch system. In the region of parameter space where the 
one-patch-one-pool combination has a locally stable equilibrium, the equilibrium of the spatial model can be either stable or unstable. Thus, with local pools, the transition from a single patch to a multi-patch situation actually decreases the range of parametervalues where the equilibrium is locally stable. Allen (1976) describes how in a system of coupled Lotka-Volterra patches the equilibrium can be destabilised when there are large differences between the dispersal rates of predators and prey. Rohani et al. (1996) discuss this effect for discrete-time models of the Nicholson-Bailey type. However, as noted already by Allen (1975), it is possible that the instability that arises due to spatial interactions is only "local" in the sense of the local stability analysis and that the formerly stable equilibrium is replaced by a stable limit cycle. Our bifurcation analysis suggests that this is indeed the case. In the range of parameter values that we investigated the stable equilibrium of the single-patch case was replaced by a stable limit cycle in the spatial case.

The population dynamics of the multi-patch system with local dispersal become more complicated in the region of parameter space where the one-patch model has an unstable equilibrium. Our simulations indicate that there are regions of parameter-space where the densities can fluctuate without going extinct. In these regions, hosts and parasitoids can coexist through the spatial differences in the system (Fig. 6). Our simulations were performed for only two patches. If further patches are added to the system, we expect the dynamics to become even more complicated and to give rise to spatial patterns such as the ones described for discrete-time models (Hassell et al. 1991b). Jansen (1994, 1995b) describes in more detail the complex dynamics that arise out of the interactions between unstable dynamics within the patches and migration between patches for a predator-prey system without pools. Note that the bounded fluctuations that arise in a spatial model should not be confused with permanence in the mathematical sense. Permanence requires that if both populations are present at the start then after some time some sizeable amount of each will be present, and this should hold for all conditions in which the initial densities are positive (Hofbauer \& Sigmund, 1988). For ecological systems, permanence is therefore a much more useful concept than local stability. Unfortunately, when a system is not permanent, as is the case in our models with a type II functional response, one would like to have information about the size of the domains of attraction, which is difficult to get.

To summarise, our spatially explicit models show a range of behaviours. In making the transition from the metapopulation model with a global pool of dispersers to a truly spatial model with local disperser pools we see a general increase in the complexity of the dynamics. The most important new property of the spatially explicit model is the emergence of spatially inhomogeneous solutions through symmetry-breaking bifurcations. Although the domains of attractions of these solutions appear to be tiny in the two-patch case investigated, they open the door for the coexistence of hosts and parasitoids.

Pools of some kind will appear in almost all biological systems. Our paper suggests that such pools and the associated cost of travelling have a stabilising influence on population dynamics. Furthermore, the redistribution of individuals over space can introduce interesting spatial dynamics. Our analysis emphasises that the details of the dispersal process determine the exact nature of host-parasitoid dynamics.

\section{REFERENCES}

Allen, J. C. (1976). Mathematical models of species interactions in space and time. Am. Nat. 109, 319-342.

Ayal, Y. \& GreEn, R. F. (1993). Optimal egg distribution among host patches for parasitoids subject to attack by hyperparasitoids. Am. Nat. 141, 120-138.

Charnov, E. L. (1976). Optimal Foraging, the Marginal Value Theorem. Theor. Pop. Biol. 9, 129-136.

Chesson, P. L. \& Murdoch, W. W. (1986). Aggregation of risk: relationships among host-parasitoid models. Am Nat. 127, 696-715.

Diekman, O., Metz, J. A. J. \& Sabelis, M. W. (1988). Mathematical models of predator/prey/plant interactions in a patch environment. Exp. Appl. Acarol., 5, 319-342.

Driessen, G. \& HemeriK, L. (1992). The time and egg budget of Leptopilina clavipes, a parasitoid of larval Drosophila. Ecol. Entomol. 17, 17-27.

GyllenberG, M. \& Hanski, I. (1992). Single species metapopulation dynamics - a structured model. J. theor. Biol. 42, 35-61.

Hamilton, W. D. \& MaY, R. M. (1977). Dispersal in stable habitats. Nature, Lond. 269, 578-581.

Hassell, M. P. (1978). The Dynamics of Arthropod Predator-prey Systems. Princeton: Princeton University Press.

Hassell, M. P. \& May, R. M. (1974). Agregation of predators and insect parasitoids and its effect on stability. J. Anim. Ecol. 43, $567-594$

Hassell, M. P., Pacala, S., May, R. M. \& Chesson, P. L. (1991a). The persistence of host-parasitoid associations in patchy environments. I. A general criterion. Am. Nat. 138, 568-583.

Hassell, M. P., Comins, H. N. \& May, R. M. (1991b). Spatial structure and chaos in insect population dynamics. Nature, Lond. 353, 255-258.

Hofbauer, S. \& Sigmund, K. (1988). The Theory of Evolution and Dynamical Systems. Cambridge: Cambridge University Press.

Hastings, A. (1983). Can spatial variation alone lead to selection for dispersal? Theor. Pop. Biol. 24, 244-251.

Holt, R. D. (1984). Spatial heterogeneity, indirect interactions, and the coexistence of prey species. Am. Nat. 124, 377-406.

Holt, R. D. (1985). Population dynamics in two-patch environments: some anomalous consequences of an optimal habitat distribution. Theor. Pop. Biol. 28, 181-208.

Holt, R. D. (1993). Ecology at the mesoscale: the influence of regional processes on local communities. In: Species Diversity in Ecological Communities (Rickleffs, R. E. \& Schluter, D. eds), pp. 77-88, Chicago: The University of Chicago Press. 
Houston, A. I. \& McNamara, J. M. (1986). The influence of mortality on the behaviour that maximizes reproductive success in a patchy environment. Oikos 47, 267-274.

HufFaKer, C. B. (1958). Experimental studies on predation: dispersion factors and predator-prey oscillations. Hilgardia 27, 343-383.

Jansen, V. A. A. (1994). Theoretical aspects of metapopulation dynamics. Ph.D. Thesis, Leiden University, Leiden, The Netherlands.

JANSEN, V. A. A. (1995a). The effects of dispersal in a tri-trophic metapopulation nodel. J. Math. Biol. 34, 195-224.

JANSEN, V. A. A. (1995b). Regulation of predator-prey systems through spatial interactions: a possible solution to the paradox of enrichment. Oikos 74, 384-390.

JANSEN, V. A. A. (1998). Local stability analysis of spatially homogeneous solutions in multi-patch systems. J. Math. Biol. (in press).

Jansen, V. A. A. \& De Roos, A. M. (1998). Predator-prey dynamics across spatial scales: the role of space in reducing population cycles. In: The Geometry of Ecological Interactions: Simplifying Spatial Complexity. (Dieckmann, U., Law, R. \& Metz, J. A. J., eds). Cambridge: Cambridge University Press.

Jansen, V. A. A. \& Sabelis, M. W. (1992). Prey dispersal and predator persistence. Exp. Appl. Acarol. 14, 215-231.

Khibnik, A. I., Kuznetsov, Y. A., Levitin, V. V. \& Nikolaev, E. V. (1993). Continuation techniques and interactive software for bifurcation analysis of ODEs and iterated maps. Physica D 62 , 360-371.

Metz, J. A. J. \& Diekman, O. (1986). The Dynamics of Physiologically Structured Populations. Vol. 96 of Lect. Notes in Biomathematics, Berlin: Springer.

Mclaughlin, J. F. \& Roughgarden, J. (1993). Species interactions in space. In: Species Diversity in Ecological Communities (Rickleffs, R. E. \& Schluter, D. eds), pp. 89-98, Chicago: The University of Chicago Press.

Murdoch, W. W. (1994). Population regulation in theory and practice. Ecology 75, 271-287.

Murray, J. D. (1975). Non-existence of wave solutions for the class of reaction diffusion equations given by the Volterra interacting-population equations with diffusion. J. theor. Biol. 52, 459-469.

Pimentel, D., Nagel, W. P. \& Maddel, J. L. (1963). Space-time structure of the environment and the survival of parasite-host systems. Am. Nat. 97, 141-168.

Pacala, S., Hassell, M. P. \& May, R. M. (1990). Host-parasitoid associations in patchy environments. Nature, Lond. 344, $150-153$.

ReEve, J. D. (1988). Environmental variability, migration, and persistence in host-parasitoid systems. Am. Nat. 132, 810-836.

ReEve, J. D. (1990). Stability, variability, and persistence in host-parasitoid systems. Ecology 71, 422-426.

Rinaldi, S., Dalbo, S. \& De NitTis, E. (1996). On the role of body size in a tri-trophic metapopulation model. J. Math. Biol. 35, 158-176.

Rohani, P., May, R. M. \& Hassell, M. P. (1996). Metapopulations and equilibrium stability: the effects of spatial structure. $J$. theor Biol. 181, 97-109.

Rosenzweig, M. L. \& MacArthur, R. H. (1963). Graphical representation and stability conditions for predator-prey interactions. Am. Nat. 97, 209-223.

Ruxton, R. D., Gonzalez-Andujar, J. L. \& Perry, J. N. (1997). Mortality during dispersal stabilises local population fluctuations. J. Anim. Ecol. 66, 289-292.

Sabelis, M. W. \& Diekman, O. (1988). Overall population stability despite local extinction: the stabilizing influence of prey dispersal from predator-invaded patches. Theor. Pop. Biol. 34, 169-176

Sabelis, M. W., Diekman, O. \& Jansen, V. A. A. (1991). Metapopulation persistence despite local extinction: predatorprey patch models of the Lotka-Volterra type. Biol. J. Linn. Soc. 42, 267-283.

Stephens, D. W. \& Krebs, J. R. (1986). Foraging Theory. Princeton: Princeton University Press.
TAYlOR, A. D. (1990). Metapopulations, dispersal and predatorprey dynamics: an overview. Ecology 71, 429-433.

TAYLOR, A. D. (1991). Studying metapopulation effects in predator-prey systems. Biol. J. Linn. Soc. 42, 305-323.

Volterra, V. (1926). Variazoni e fluttuazioni d'individui in specie animali conviventi. Mem. Acad. Lincei 2, 31-113. (Translated in Scudo, F. M. \& Zeigler, J. R. (1978) The Golden Age of Theoretical Ecology: 1923-1940. Lect. Notes in Biomath. 22 pp. 65-237. Berlin, Springer).

Weisser, W. W. \& Hassell, M. P. (1996). Animals 'on the move' stabilise host-parasitoid systems. Proc. R. Soc. Lond. B 263, 749-754.

Weisser, W. W., Houston, A. I. \& VöLkL, W. (1994). Oviposition strategies of solitary parasitoids: The trade-off between female and offspring mortality risks. Evol. Ecol. 8, 587-597.

Weisser, W. W., Wilson, H. B. \& Hassell, M. P. (1997). Interference among parasitoids: a clarifying note. Oikos 79, 173-178.

\section{APPENDIX A}

In this section we show that system (1) has a unique equilibrium which is globally stable. To do this we define the function

$$
v_{j(t)}=H_{j}(t)+P_{j}(t)-H^{*} \ln H_{j}-P^{*} \ln P_{j}(t) .
$$

The function $V(t)$ defined as:

$$
\begin{gathered}
V(t)=\frac{\eta}{n \eta+\sigma} R(t)-\frac{n \eta \varepsilon H^{*}}{(n \eta+\sigma)^{2}} \ln R(t)+\frac{i}{n i+s} Q(t) \\
-\frac{n e i P^{*}}{(n i+s)^{2}} \ln Q(t)+\sum_{j=1}^{n} \frac{v_{j}(t)}{n}
\end{gathered}
$$

is positive definite when the equilibrium is translated to the origin. We will show that $V(t)$ is a Lyapunov function for (1). This can be shown by writing out the derivative of $V$ with respect to time:

$$
\begin{gathered}
\dot{V}=\frac{\eta}{n \eta+\sigma} \dot{R}-\frac{n \eta \varepsilon H^{*}}{(n \eta+\sigma)^{2}} \frac{\dot{R}}{R} \\
+\frac{i}{n i+s} \dot{Q}-\frac{n e i P^{*}}{(n i+s)^{2}} \frac{\dot{Q}}{Q}+\sum_{j=1}^{n} \frac{\dot{v}_{j}}{n} \\
=-\frac{\eta H^{*}}{n} \sum_{j=1}^{n} \frac{\left(R-\frac{n \varepsilon}{n \eta+\sigma} H_{j}\right)^{2}}{R H_{j}} \\
-\frac{i P^{*}}{n} \sum_{j=1}^{n} \frac{\left(Q-\frac{n e}{n i+s} P_{j}\right)^{2}}{Q P_{j}} \leqslant 0
\end{gathered}
$$

Thus, all $\omega$-limits of system (1) must lie contained in the set $\left\{H_{j}, P_{j}, R, Q \in \mathbb{R}^{2(n+1)} \mid \dot{V}=0\right\}$ which is identical to $\left\{H_{j}, P_{j}, R, Q \in \mathbb{R}^{2(n+1)} \mid(n \eta+\sigma) R=n \varepsilon H_{j}\right\} \cap\left\{H_{j}, P_{j}\right.$, $\left.R, Q \in \mathbb{R}^{2(n+1)} \mid(n i+s) Q=n e P_{j}\right\}$. Clearly, within this 
intersection of sets all $H_{j}$ are identical and all $P_{j}$ are identical and therefore

$$
\dot{R}=\varepsilon \sum_{j=1}^{n} H_{j}-(n \eta+\sigma) R=\varepsilon n H_{j}-(n \eta+\sigma) R=0
$$

and

$$
\dot{Q}=e \sum_{j=1}^{n} P_{j}-(n i+s) Q=e n P_{j}-(n i+s) Q=0 .
$$

Hence all variables are constant within the intersection. The only invariant subset is the interior equilibrium. In any other equilibrium the derivative of the Lyapunov function must be zero. Since the derivative of the Lyaponuv function is zero in a single equilibrium this equilibrium must therefore be unique.

When one of the species, say the predators, does not disperse, the $\omega$-limit set lies contained in $\left\{H_{j}, P_{j}\right.$, $\left.R, Q \in \mathbb{R}^{2(n+1)} \mid(n \eta+\sigma) R=n \varepsilon H_{j}\right\}$. In this set all $H_{j}$ are equal and $R$ is constant. The only invariant subset in the interior is the interior equilibrium. Note that other equilibria exist, for which the predator density is zero, but that none of these equilibria is stable.

\section{APPENDIX B}

In this section we analyse the stability of the equilibrium of system (3) where each patch is surrounded by their local pool of dispersers. For the purpose of the present paper, we only analyse the case of two patches connected by dispersal via their local pools. An extensive description of how to derive the stability criteria for an arbitrary number of patches with an arbitrary connectivity matrix $\mathbf{C}$ will be published elsewhere (Jansen, 1998). In the case of two patches,

$$
\mathbf{C}=\left(\begin{array}{rr}
-1 & 1 \\
1 & -1
\end{array}\right)
$$

Because the population dynamics in both patches are governed by identical equations with identical parameters there exists an equilibrium in which the densities in both patches are the same, i.e. $H_{1}^{*}=H_{2}^{*}=H^{*}, P_{1}^{*}=P_{2}^{*}=P^{*}, R_{1}^{*}=R_{2}^{*}=R^{*}$ and $Q_{1}^{*}=Q_{2}^{*}=Q^{*}$. For the analysis of stability, we transform the variables of the system into four variables that describe the main densities: $H=\frac{1}{2}\left(H_{1}+H_{2}\right), \quad P=\frac{1}{2}\left(P_{1}+P_{2}\right), \quad R=\frac{1}{2}\left(R_{1}+R_{2}\right)$, $Q=\frac{1}{2}\left(Q_{1}+Q_{2}\right)$, and four variables describing the differences between the patches: $\Delta_{H}=\frac{1}{2}\left(H_{1}-H_{2}\right)$, $\Delta_{P}=\frac{1}{2}\left(P_{1}-P_{2}\right), \quad \Delta_{R}=\frac{1}{2}\left(R_{1}-R_{2}\right), \quad \Delta_{Q}=\frac{1}{2}\left(Q_{1}-Q_{2}\right)$. For analysis of stability we analyse the equations in the neighbourhood of $\Delta_{H}=\Delta_{P}=\Delta_{R}=\Delta_{Q}=0$. In the linearized system the dynamics of $H, P, R$, and $Q$ are given by system (3) (with $m_{q}=m_{r}=0$ ). The dynamics of the other four variables are given by

$$
\begin{gathered}
\dot{\Delta}_{H}=(r-P-\varepsilon) \Delta_{H}-H \Delta_{P}+\eta \Delta_{R} \\
\dot{\Delta}_{P}=P \Delta_{H}+(H-d-e) \Delta_{P}+i \Delta_{Q} \\
\dot{\Delta}_{R}=\varepsilon \Delta_{H}-\left(\eta+\sigma+2 m_{r}\right) \Delta_{R} \\
\dot{\Delta}_{Q}=e \Delta_{P}-\left(i+s+2 m_{q}\right) \Delta_{Q}
\end{gathered}
$$

Note that these equations can be written as $\dot{\Delta}=(\mathbf{J}(\mathbf{S})+\lambda \mathbf{M}) \Delta$, where $\boldsymbol{\Delta}=\left(\Delta_{H}, \Delta_{P}, \Delta_{R}, \Delta_{Q}\right)^{T}$, $\mathbf{S}=(H, P, R, Q)^{T}$, and $\mathbf{J}$ is the Jacobian of system (3) with $m_{q}=m_{r}=0$, evaluated at $\mathbf{S}$. The matrix $\mathbf{M}$ is the $4 \times 4$ matrix where all entries are zero except for $m_{33}=m_{r}$ and $m_{44}=m_{q}$. The parameter $\lambda$ is a non-zero eigenvalue of $\mathbf{C}$. In our case $\lambda=-2$.

To assess the local stability of the equilibrium of system (3) it now suffices to analyse system (3) with $m_{q}=m_{r}=0$ (this system is always stable) and system (B.2) at $\mathbf{S}^{*}=\left(H^{*}, P^{*}, R^{*}, Q^{*}\right)^{T}$. The Jacobian of the system (B.2) is given by

$$
\left[\begin{array}{cccc}
\frac{-\varepsilon \eta}{\eta+\sigma}-H^{*} & \eta & 0 \\
P^{*} & \frac{-i e}{i+s} & 0 & i \\
\epsilon & 0 & -\left(\eta+\sigma+2 m_{r}\right) & 0 \\
0 & e & 0 & -\left(i+s+2 m_{q}\right)
\end{array}\right)
$$

which can be rewritten as

$$
\left(\begin{array}{cccc}
-a_{11} & -a_{12} & a_{13} & 0 \\
a_{21} & -a_{22} & 0 & a_{24} \\
a_{31} & 0 & -a_{34}-2 m_{r} & 0 \\
0 & a_{42} & 0 & -a_{44}-2 m_{q}
\end{array}\right)
$$

with $a_{11}=a_{13} a_{31} / a_{33}$ and $a_{22}=a_{24} a_{42} / a_{44}$. The analysis of the fourth-order characteristic equation of this matrix results in rather long algebraic equations. Implementing this in a program for formulae manipulation is straightforward and reveals that all eigenvalues of the matrix are negative. Thus, the equilibrium of the two-patch system is always locally asymptotically stable. For a multi-patch system where $\mathbf{C}$ is different, local stability of the equilibrium can be assessed in essentially the same way. The only difference is that $\lambda$ can now take values different from -2 . In general, once the stability of the two-patch system is known, the analysis of the stability of the equilibrium (or any other attractor) is reduced to finding the eigenvalues of $\mathbf{C}$ and performing a scaling operation (Jansen, 1998). 


\section{APPENDIX C}

In this section, we give the stability criteria for system (5). The coefficients of the characteristic equation $\sum_{i=0}^{3} a_{i} \lambda^{i}$ are:

$$
\begin{aligned}
& a_{0}=\alpha r\left(1-\alpha T_{h}\right)(i+s) \\
& a_{1}=\alpha r\left(1-T_{h}(i+s+d+e)\right) \\
& a_{2}=(i+s+d+e)-\alpha-r \alpha T_{h} \\
& a_{3}=1
\end{aligned}
$$

The Routh-Hurwitz conditions for local asymptotic stability are $a_{0}>0, a_{2}>0$, and $a_{1} a_{2}>a_{0}$. This first condition is automatically fulfilled if the equilibrium is positive. The second condition requires

$$
r<\frac{1}{\alpha T_{h}}\left(i+s+\frac{i e}{i+s}\right) .
$$

The third conditions requires $r>f\left(T_{h}\right)$ if $T_{h}>$ $(i+s+d+e)^{-1} \quad$ and $\quad r<f\left(T_{h}\right) \quad$ if $\quad T_{h}<$ $(i+s+d+e)^{-1}$ where

$$
\begin{aligned}
& f\left(T_{h}\right)= \\
& \quad \frac{1}{\alpha T_{h}}\left(i+s+\frac{i e}{i+s}-\frac{\left(1-\alpha T_{h}\right)(i+s)}{1-T_{h}(d+e+i+s)}\right)
\end{aligned}
$$

When $T_{h}>(i+s+d+e)^{-1}$ condition (C.2) and $r>f\left(T_{h}\right)$ cannot be fulfilled simultaneously so that $T_{h}<(i+s+d+e)^{-1}$ and $r<f\left(T_{h}\right)$ are necessary and sufficient conditions for the positive equilibrium to be locally asymptotically stable. These are the conditions given in (7). Note that it is only necessary to know five of the six parameters.

\section{APPENDIX D}

Here, we analyse the stability criteria for system (8). We transform to the new variables $H, P, \Delta_{H}$ and $\Delta_{P}$ as defined in Appendix $\mathrm{B}$ and linearise around $\Delta_{H}=\Delta_{P}=0$ to arrive at the decoupled systems (5) and:

$$
\begin{gathered}
\dot{\Delta}=\left(r-\frac{P}{\left(1+T_{h} H\right)^{2}}\right) \Delta_{H}-\frac{H}{\left(1+T_{h} H\right)^{2}} \Delta_{P} \\
\dot{\Delta}_{P}=\frac{P}{\left(1+T_{h} H\right)^{2}} \Delta_{H}+\left(\frac{H}{\left(1+T_{h} H\right)^{2}}-d-e\right) \Delta_{P}
\end{gathered}
$$

Note that this can be written as $\dot{\boldsymbol{\Delta}}=(\mathbf{J}(\mathbf{S})-\mathbf{E}) \boldsymbol{\Delta}$, where $\mathbf{S}=(H, P)^{T}$, and the matrix $\mathbf{E}$ is the $2 \times 2$ matrix where all entries are zero except for $e_{22}=e$. One equilibrium of the system is given by $\mathbf{S}^{*}=\left(H^{*}\right.$, $\left.P^{*}\right)^{T}, Q=Q^{*}, \Delta_{H}=\Delta_{P}=0$, i.e. the densities of hosts and parasitoids in both patches are identical. From the equations it is clear that the subspace $\Delta_{H}=\Delta_{P}=0$ is invariant. The Jacobian of system (D.1) at $\mathbf{S}^{*}$ is:

$$
\left(\begin{array}{cc}
\alpha r T_{h} & -\alpha \\
r-\alpha r T_{h} & \alpha-d-e
\end{array}\right)
$$

There is a Hopf bifurcation at

$$
r=\frac{1}{T_{h} \alpha} \frac{i e}{i+s} .
$$

The determinant is zero (one eigenvalue equal to zero) at $T_{h}=(d+e)^{-1}$. Comparison with conditions (7) shows that none of these conditions can be fulfilled while the equilibrium of (5) is asymptotically stable. Figure 3 shows an example of a numerical bifurcation analysis. Indeed, none of the new bifurcations occur on the stable equilibrium.

\section{APPENDIX E}

In this final appendix we analyse the case when dispersal is into local pools and the parasitoids have a Holling type II functional response. For simplicity, we assume that hosts are sessile and only parasitoids are dispersing between patches.

We proceed as in Appendix B and analyse the two-patch case first. After transforming the system and linearising as before, we arrive at system (9) and the remaining equations are given as $\dot{\Delta}=\mathbf{D} \Delta$ where $\boldsymbol{\Delta}=\left(\Delta_{H}, \Delta_{P}, \Delta_{Q}\right)^{T}$. The matrix $\mathbf{D}$ is given by

$$
\left(\begin{array}{ccc}
r-\frac{P}{\left(1+T_{h} H\right)^{2}} & \frac{-H}{1+T_{h} H} & 0 \\
\frac{P}{\left(1+T_{h} H\right)^{2}} & \frac{H}{1+T_{h} H}-d-e & i \\
0 & e & -\left(i+s+2 m_{q}\right)
\end{array}\right)
$$

At equilibrium, the Jacobian is given by

$$
\left(\begin{array}{ccc}
\alpha r T_{h} & -\alpha & 0 \\
r-\alpha r T_{h} & \alpha-d-e & i \\
0 & e & -\left(i+s+2 m_{q}\right)
\end{array}\right)
$$


The coefficients of the characteristic equation $\sum_{i=0}^{3} a_{i} \lambda^{i}$ are

$$
\begin{aligned}
& a_{0}=\alpha r\left(2 m_{q} T_{h}(\alpha-d-e)+\left(1-\alpha T_{h}\right)\left(i+s+2 m_{q}\right)\right. \\
& a_{1}=-(\alpha-d-e) 2 m_{q} \\
& \quad \quad-\alpha r\left(T_{h}\left(i+s+2 m_{q}+d+e\right)-1\right) \\
& a_{2}=i+s+2 m_{q}+d+e-\alpha\left(1+r T_{h}\right) \\
& a_{3}=1
\end{aligned}
$$

The equilibrium is unstable if $a_{1} a_{2}<a_{0}$ (the other Routh-Hurwitz criteria are always fulfilled) which is when:

$$
A\left(2 m_{q}\right)^{2}+B\left(2 m_{q}\right)+C>0
$$

where

$$
\begin{gathered}
A=\alpha-d-e+\alpha r T_{h} \\
B=A(i+s-A)+\alpha r T_{h}(i+s) \\
C=\alpha r\left[\left(T_{h}(i+s+d+e)-1\right)(i+s-A)\right. \\
\left.+\left(1-\alpha T_{h}\right)(i+s)\right] .
\end{gathered}
$$

Note that the non-spatial system is stable if $C<0$. Spatial interaction can only destabilise the equi- librium if $A$ and $B$ differ in sign, which happens, for instance, for the parameter combination used in Fig. 5. Thus, in the case of patches connected by local pools, the equilibrium of the spatial system is locally asymptotically stable for a smaller range of parameter values than the non-spatial system. In the range of parameter-values where the equilibrium of the single patch is locally asymptotically stable, the Hopf-bifurcations give rise to asymptotically stable limit cycles. If simulations are started outside the domain of attraction of either the asymptotically stable equilibrium or the limit cycle, the population densities of hosts and parasitoids quickly go to infinity. Thus, in the range of parameter-values where the equilibrium of the single patch with its pool was locally asymptotically stable, two identical patches with local pools are not more permanent than a single patch with its pool. In contrast, in the region of parameter-space where the single-patch case was unstable, the dynamics of the two patches connected via local pools are even more complicated than for the case of two-patches connected by a global pool (Appendix D). In our simulations we found bounded fluctuations in a small region of parameter space. Figure 5 gives an example of such a simulation. 